\title{
Archéopages
}

Archéopages

Archéologie et société

\section{Les animaux en ville. Des squelettes médiévaux et modernes à Amiens}

\section{Benoît Clavel et Opale Robin}

\section{(2) OpenEdition}

1 Journals

Édition électronique

URL : https://journals.openedition.org/archeopages/299

DOI : 10.4000/archeopages.299

ISSN : 2269-9872

\section{Éditeur}

INRAP - Institut national de recherches archéologiques préventives

\section{Édition imprimée}

Date de publication : 1 mai 2013

Pagination : 48-51

ISSN : $1622-8545$

\section{Référence électronique}

Benoît Clavel et Opale Robin, « Les animaux en ville. Des squelettes médiévaux et modernes à Amiens

», Archéopages [En ligne], 35 | 10/2012, mis en ligne le 01 octobre 2014, consulté le 22 janvier 2022.

URL : http://journals.openedition.org/archeopages/299; DOI : https://doi.org/10.4000/archeopages. 299

(c) Inrap 


\section{Les animaux en ville \\ Des squelettes médiévaux et modernes à Amiens}

Benoît Clavel CNRS, UMR 7209 "Archéozoologie, archéobotanique : sociétés, pratique et environnement"

Opale Robin CRAVO, universitéde Poitiers

48

1. Responsable

d'opération Vincent

Lascourt, Inrap.
La fouille préventive du site d'Amiens (Somme) «Avenue Charles de Gaulles » ${ }^{\mathbf{1}}$ a mis au jour un îlot urbain dont les vestiges s'échelonnent du haut Moyen-Âge à la période moderne. Celle-ci a livré, notamment, des squelettes complets ou quasi complets de grand et de petit bétail, enfouis individuellement, au sein ou parfois en recoupement de constructions et de fosses. L'étude de cette zone archéologique vise à comprendre la gestion des déchets animaux en milieu urbain. La découverte de tels dépôts pose la question de la présence d'une activité liée à lélevage au cœur ou en périphérie de la ville d'Amiens à la Renaissance.

L'ensemble de la faune étudiée est issue de cinq unités stratigraphiques et concerne 978 restes collectés pour une masse totale de $18,8 \mathrm{~kg}$ [ill. 1]. On notera la présence exclusive des bœufs, porcs et caprinés, auxquels il faut ajouter quelques ossements qui n'ont pu être rattachés à un groupe spécifique. De prime abord, le décompte de chaque pièce osseuse semble suggérer l'existence d'un grand nombre de vestiges animaux. En réalité les ossements se répartissent en quelques ensembles anatomiques. La particularité de ce corpus réside donc dans la présence de squelettes en connexion témoignant de la mortalité animale au sein de cet îlot urbain. Ainsi, par exemple,

le rejet de quatre cadavres de cochons, une truie et trois porcelets, dans l'US 4189 illustre probablement des conditions d'élevage regrettables [ill. 2].

Il n'est pas impossible que les porcelets soient issus de la même portée. L'estimation de lâge de nos trois petits cochons a été réalisée à partir des mesures des os longs. En effet, d'après Karl-Heinz Habermehl (1975), une correspondance est possible entre la longueur des os de porcs juvéniles et leur âge. Globalement, les longueurs totales des diaphyses des jeunes cochons s'accordent avec des individus âgés entre 96 et 115 jours ( 3 et 4 mois). Il s'agit donc probablement de suidés morts peu de temps après leur naissance et issus de la même portée. Concernant l'animal adulte, lâge a été estimé à partir des dents. Chaque usure dentaire a été notée d'après des codes spécifiques établis par A. Grant (1976). Deux auteurs proposent d'associer ces codes à des âges de cochon : Peter RowleyConwy (1993) et Marie-Pierre Horard-Herbin (1997). Seuls les travaux de cette dernière sont assez précis pour permettre d'estimer l'âge de l'animal entre 16 et 20 mois. D'après ces observations, la truie avait donc atteint sa maturité sexuelle et pouvait potentiellement être la mère des jeunes cochons. Le Théâtre d'agriculture et mesnage des champs d'Olivier de Serres (16oo, p. 333-334) précise que la truie peut être saillie dès ses cinq mois : «Au cinquiefme mois, cochonne la truye, aiant cela de particulier, que de fe remplir deux fois l'année... »

Il n'a, en revanche, pas été possible de définir la véritable cause de la mort de ces animaux. Néanmoins, si la découverte de squelettes en connexion écarte l'hypothèse d'une consommation de ces bêtes, les traces de découpes témoignent d'une manipulation du cadavre de la truie (ouverture du thorax et prélèvement de sous-produits) [ill. 3]. D'autres contextes ont livré des restes de cadavres, comme l'US 4216 contenant une truie âgée d'environ 2 ans exempte de toute trace d'intervention, ou bien encore l'US 4192 avec, en dépôt, un verrat de moins de 20 mois, une truie de plus 42 mois et un mouton de 6 à 8 ans. Pour finir, évoquons la présence d'une vache de réforme non loin de là, dans la couche 4193 [ill. 4].

La présence de ces cadavres peut être due à de nombreux facteurs qu'il ne nous est pas vraiment possible de départager : les maladies, 


\begin{tabular}{|c|c|c|c|c|c|c|c|}
\hline \multicolumn{2}{|c|}{$\begin{array}{c}\text { Amiens } \\
\text { «Avenue Charles de Gaulles » }\end{array}$} & NR & $\%$ NR & Masse (g) & \% Masse & $\begin{array}{c}\text { Masse } \\
\text { moyenne (g) }\end{array}$ & \multirow{9}{*}{$\begin{array}{l}\text { 1. Dénombrements de } \\
\text { restes, période moderne, } \\
\text { Amiens « Avenue Charles } \\
\text { de Gaulle I: } \\
\text { NR, un ensemble } \\
\text { anatomique = 1 reste; } \\
\text { poids PR, en g; } \\
\text { poids moyen PM, en g; } \\
\text { *n= présence de n } \\
\text { ensembles anatomiques } \\
\text { par espèce. } \\
2 . \text { Squelette de la truie } \\
\text { et des porcelets (cercles } \\
\text { jaunes) en connexion lors } \\
\text { de la fouille. }\end{array}$} \\
\hline Bœuf & Bos taurus & $8 * 1$ & 15,7 & 8119 & 43,3 & 1014,9 & \\
\hline Porc & Sus scrofa domesticus & $18 * 7$ & 35,3 & 10111 & 54,0 & 561,7 & \\
\hline Caprinés & Caprini & $25 * 1$ & 49 & 504 & 2,7 & 20,2 & \\
\hline \multicolumn{2}{|c|}{ Total des ensembles déterminés } & 51 & 65,4 & 18734 & 99,7 & 367 & \\
\hline \multicolumn{2}{|c|}{ Indéterminés grand bétail } & 7 & 9 & 47 & 0,3 & 6,7 & \\
\hline \multicolumn{2}{|c|}{ Indéterminés petit bétail } & 7 & 9 & 6 & - & 0,9 & \\
\hline \multicolumn{2}{|c|}{ Indéterminés } & 13 & 16,7 & 12 & 0,1 & 0,9 & \\
\hline \multicolumn{2}{|c|}{ Total mammifères } & 78 & & 18799 & & 241 & \\
\hline
\end{tabular}

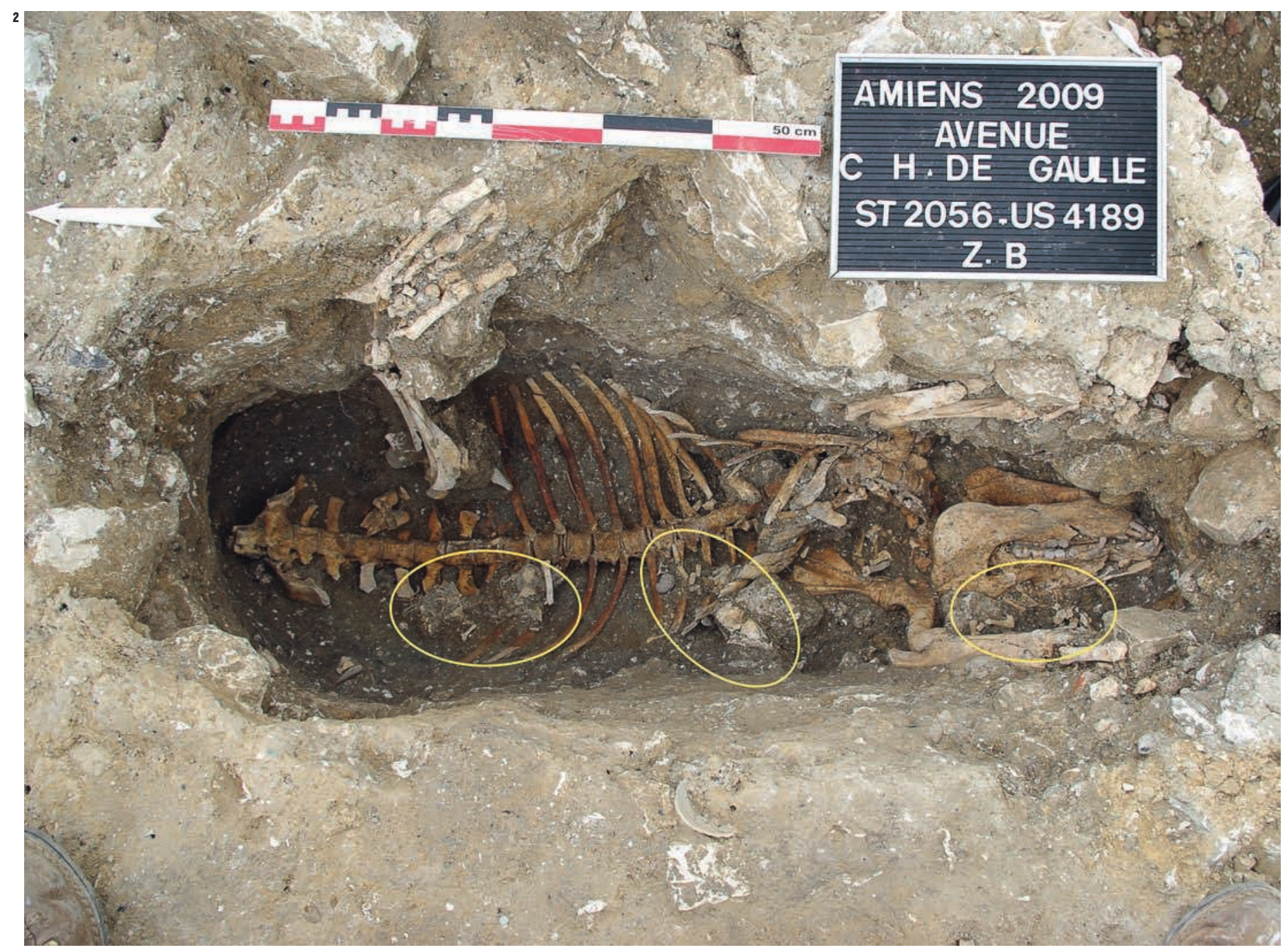


la malchance, l'approximation dans les techniques d'élevage et probablement des conditions d'élevage quelquefois désastreuses (manque d'espace, mauvaise hygiène) sont autant de causes de mortalités. Les problèmes de contamination dans la société médiévale et moderne se posent au quotidien. Les produits animaux peuvent même être une source de contaminations indirectes, comme le cuir non tanné (peste bovine) ou la laine (fièvre charbonneuse pour les ovins). Dans un élevage, l'hygiène doit être la règle et le contrôle de la population des rongeurs (les rats) comme des insectes (vecteurs de virus) est indispensable. En ville, l'entretien régulier de la fumière ou l'évacuation des lisiers est plus difficile qu'à la campagne. Les bâtiments plus ou moins bien entretenus favorisent donc par exemple le développement des larves et génèrent une multiplication de cohortes d'insectes. Celles-ci (adjointes à bien d'autres facteurs) peuvent être à l'origine d'épizooties ou d'une mortalité frappant certains animaux (jeunes truies allaitantes, animaux de réforme moins résistants). Des sources littéraires médiévales nous renseignent sur cet aspect particulier des animaux malades et sur leur devenir. Certains ouvrages réglementaires, religieux par exemple, comme les pénitentiels (antérieurs, il est vrai), interdisent la consommation de ces bêtes malades, mortes et impures (Bonnassie, 1989, p. 1037). Les ouvrages réglementaires civils ou les règlements de boucherie manifestent aussi des préoccupations sanitaires vis-à-vis des animaux malades, impropres à la consommation (Ferrières, 2002).

Cette collection de squelettes plus ou moins entiers, de cochons, de bœufs et de moutons témoigne donc de l'activité d'élevage en zone urbaine ou péri-urbaine vers la fin du Moyen Âge et à la Renaissance, mais elle témoigne aussi du mode de gestion des dépouilles d'animaux isolés. Elle est d'ailleurs à rapprocher des découvertes réalisées sur le site du «Grand Vidame » ${ }^{2}$. Une petite série de trois cochons y avait été mise au jour. Ces animaux avait été ensevelis aussi dans une même parcelle après avoir été atteints d'une pathologie osseuse (non déterminée) ayant fortement altéré les os. De même, un ensemble de restes de porcs, daté du $\mathrm{XIV}^{\mathrm{e}}$ siècle et composé de pièces de foetus, de nouveaux-nés, de cochons de lait et d'une truie a été retrouvé à Paris, au Louvre, dans le quartier de la rue Fromenteau (Meniel, Arbogast, 1989). Cet ensemble témoignerait d'un événement exceptionnel, sans doute une épidémie. Mais, là encore, il s'agit d'un exemple illustrant la présence d'une porcherie dans les murs de Paris au XIV ${ }^{\mathrm{e}}$ siècle. La reproduction sur place y est attestée comme sur le site d'Amiens «Avenue Charles de Gaulle».

L'archéologie témoigne régulièrement de ces agissements, surtout en milieu rural, et notamment avec le site « Le Tordoir ${ }^{3}$ à Dourges (Nord-Pas-de-Calais) (Blanquaert et al., 2007), où plus de 11 cochons, 1 poulain, 3 chiens, 1 veau, 2 moutons et 3 coqs ont été découverts dans
13 structures datées entre le bas Moyen Âge et la période moderne. Sur le site du XIV $\mathrm{X}^{\mathrm{e}}$ siècle de la «Crônerie 2 » (Maine-et-Loire) ${ }^{\mathbf{4}}$, des bœufs ont été ensevelis en un même endroit, loin de la zone d'habitation.

À la campagne, l'évacuation des animaux morts est donc gérée au cas par cas, contrairement au monde urbain dans lequel, en règle générale, la gestion des nombreuses dépouilles animales a généré un commerce lucratif. Cela a provoqué la création de clos d'équarrissage situés à l'écart ou en périphérie du noyau urbain. Cette activité autour des dépouilles était exercée par une certaine frange de la population dont la motivation première était avant tout la récupération de la peau, mais aussi de la graisse, des tendons, des os, du crin ou de la corne pour ne citer que les principaux. Les animaux du site de l'avenue Charles de Gaulle ont été en tout état de cause ensevelis sur une même parcelle. Reste à savoir si cette zone, qui se situe dans les faubourgs de la ville, était à ce moment-là abandonnée, en friche. Les troubles de la fin du $\mathrm{XIV}^{\mathrm{e}}$ siècle ont-ils provoqué un abandon partiel des faubourgs construits de la ville? C'est ce que pourrait suggérer le creusement de la fosse 4216, contenant une truie, au beau milieu d'un enfeu de cheminée de belle facture.

Le cas de ces squelettes enterrés, probablement dans des parcelles urbaines, illustre le pragmatisme de ces citadins qui ont su tirer le meilleur parti des circonstances lorsqu'ils ont été confrontés à des événements souvent peu prévisibles et surtout difficiles. Seule la mise en perspective de ces résultats avec ceux d'autres sites de la région pourra nous aider à mieux cerner le phénomène de mortalité et d'hygiène des animaux d'élevage en ville, questions sur lesquelles les sources documentaires ne manquent pas. L'animal malade au Moyen Âge reste un thème assez peu étudié de sorte que le recensement systématique des sources archéozoologiques et l'analyse approfondie des cadavres ou des charniers demeurent les sources d'indications majeures sur ces problématiques. d'opération, Dominique

Gemehl, Inrap.

3. Responsable

d'opération, Geertrui

Blancquaert, Inrap, 2007.

4. Responsable

d'opération, Frédéric

Guérin, Inrap. 


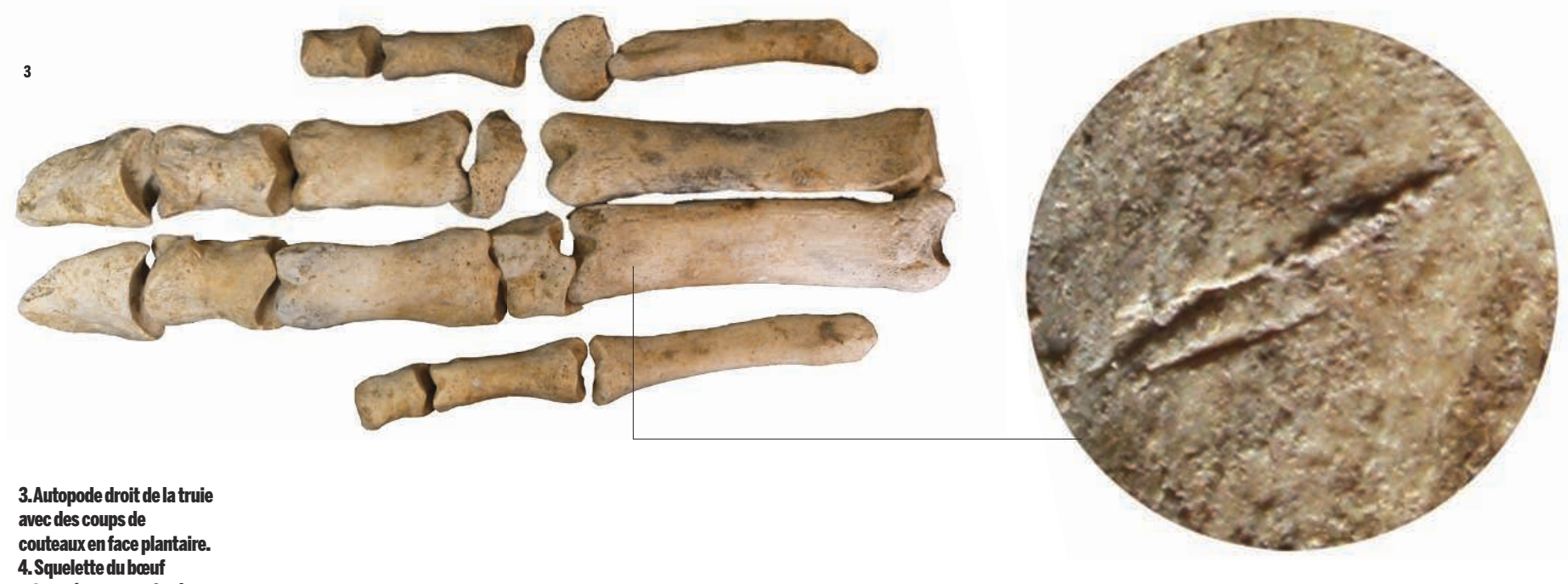

4. Squelette du beeuf

retrouvé en connexion lors

de la fouille.

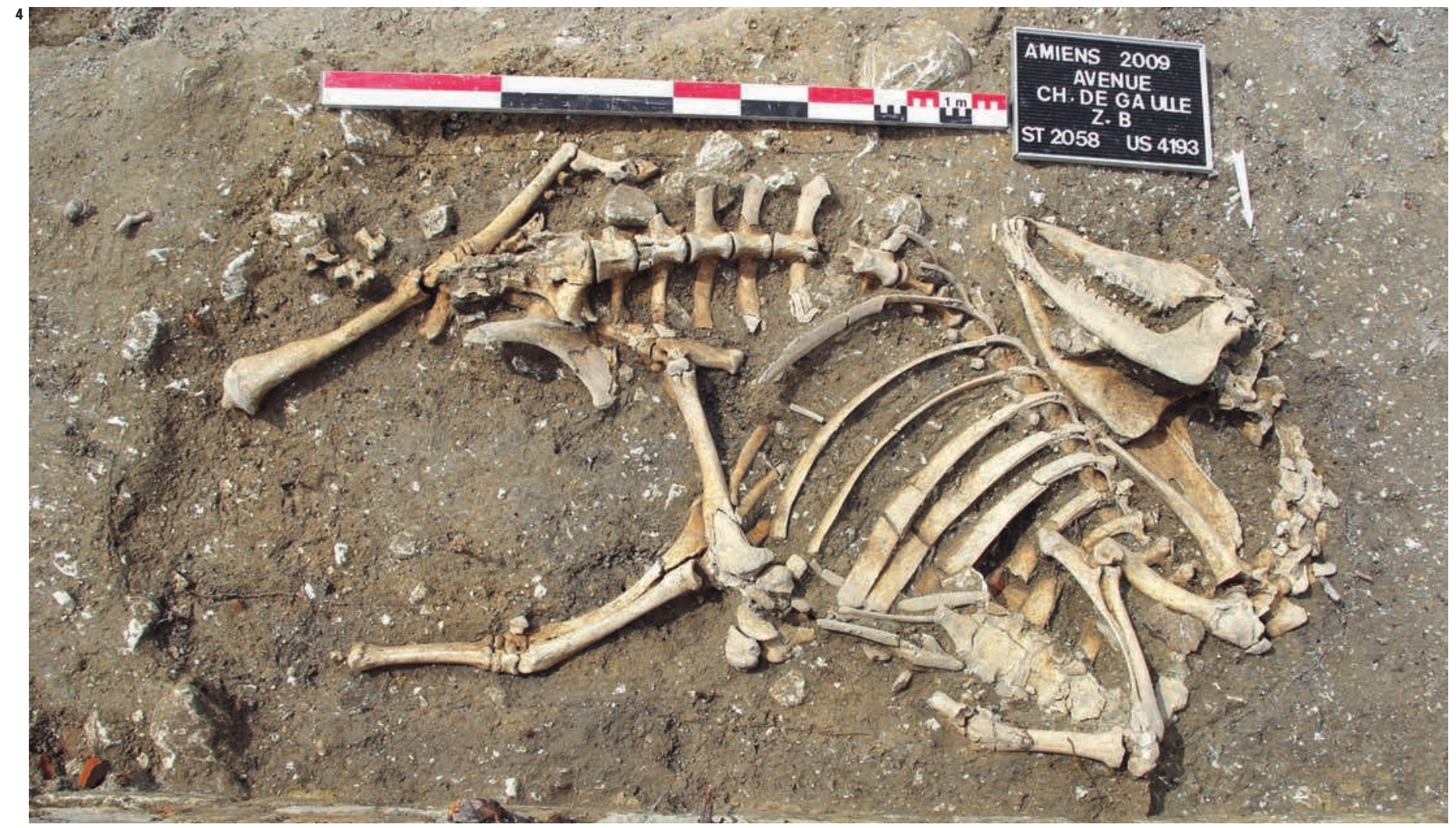

\section{Références bibliographiques}

Serres O. De, (160o), Lieutaghi P. (ÉD.), Le Théatre d'agriculture et mesnage des champs, Paris, Actes Sud, 2001, 1550 p.

BlANCQUAERT G. (DIR.), 2007, «La plate-forme multimodale Delta 3 de Dourges, premiers résultats des fouilles préventives », Gauheria, hors-série, $120 \mathrm{p}$.

BonNASSIE P., 1989, « Consommation d'aliments immondes et cannibalisme de survie dans

l'Occident du haut Moyen Âge ", Annale Economies, Sociétés, Civilisations, vol. 44, $\mathrm{n}^{\circ} 5$ (septembre-octobre) p. 1035-1056.

FERRIÈRES M Moyen Âge à l'aube du XX $X^{e}$ siècle, Paris, Seuil, $473 \mathrm{p}$.

Grant A., 1976, « The use of tooth wear as a guide the age of domestic animals, a brief explanation ", in Cunliffe B, Excavations at Portchester Castle, Reports of the Research Committee of the Society of Antiquaries of London, p. 437-450.
HABERMEHL K.H., 1975, Die alterbestimmung bei Haus-und Labortieren, Berlin et Hambourg, Paul Parey Verlag. HorARD-HERBIN M.P., 1997, «Le village celtique des Arènes à Levroux. Lélevage et les productions animales dans l'économie de la fin du second âge du Fer, Levroux 4.", Revue Archéologique du Centre de la France, suppl. 12, FERACF-ADEL, Levroux, 208 p.

Meniel P., Arbogast R.-M., 1989, « Les restes de mammifères de la cour Napoléon du Louvre (Paris) du XIV ${ }^{\mathrm{e}}$ au XVIII ${ }^{\mathrm{e}}$ siècle », Revue de Paléobiologie, vol. 8, 2, p. 405-466, 97 fig.

ROWLEY-CONWY P., 1993, «Season and reason: the case for a regional interpretation of mesolithic settlements patterns ", in Peterkin G.L., Bricker H., Mellars P., Hunting and animal exploitation in later Paleolithic and Mesolithic of Eurasia, American anthropological Association, $n^{\circ}$ 4, p. 179-188. 